\title{
New results from the OPERA experiment
}

\author{
Sergey Dmitrievsky ${ }^{1, \text { a }}$ \\ on behalf of the OPERA collaboration \\ ${ }^{1}$ Joint Institute for Nuclear Research, \\ 141980 Dubna, Moscow region, Russia
}

\begin{abstract}
The OPERA experiment reached its main goal by proving the appearance of $v_{\tau}$ in the CNGS $v_{\mu}$ beam. A total sample of 5 candidates fulfilling the analysis defined in the proposal was detected with a S/B ratio of about ten allowing to reject the null hypothesis at $5.1 \sigma$. The search has been extended to $v_{\tau}$-like interactions failing the kinematical analysis defined in the experiment proposal to obtain a statistically enhanced, lower purity, signal sample. Based on the enlarged data sample the estimation of $\Delta m_{23}^{2}$ in appearance mode is presented. The search for $v_{e}$ interactions has been extended over the full data set with a more than twofold increase in statistics with respect to published data. The analysis of the $v_{\mu} \rightarrow v_{e}$ channel is updated and the implications of the electron neutrino sample in the framework of the $3+1$ sterile model is discussed. An analysis of $v_{\mu} \rightarrow v_{\tau}$ interactions in the framework of the sterile neutrino model has also been performed. Moreover the results of the analysis of the annual modulation of the cosmic muon rate will be presented.
\end{abstract}

\section{Introduction}

The 3-flavour oscillation framework and the PMNS matrix describe with success the results from solar, atmospheric, reactor and accelerator neutrino experiments. However most of the results have been obtained from neutrino disappearance phenomena involving muon and electron neutrinos and anti-neutrinos, the only exceptions being $v_{\mu} \rightarrow v_{e}$ from T2K and NOvA. For a complete confirmation of the whole theoretical framework the oscillation picture needs to be proved also in the flavour appearance modes.

The OPERA experiment [1] was designed to detect $v_{\mu} \rightarrow v_{\tau}$ oscillations in direct $v_{\tau}$ appearance mode in an almost pure muon neutrino beam produced at CERN. The OPERA hybrid detector with a target mass of $\sim 1.25$ kton combined electronic detectors (such as scintillator strips and resistive plate chambers) to register the neutrino interaction events in real time and nuclear emulsions used as very precise tracking devices. Such a concept made possible detection of short-lived tau-leptons produced in $v_{\tau}$ charged current $(\mathrm{CC})$ interactions with a high signal-to-background ratio.

In addition to the dominant $v_{\mu} \rightarrow v_{\tau}$ oscillation, the sub-leading $v_{\mu} \rightarrow v_{e}$ transition occured as well due to the non zero $\theta_{13}$ mixing angle. This process was also investigated by OPERA

ae-mail: dmitr@jinr.ru 
profiting from its electron identification capabilities. This also allowed to study possible non standard oscillation process.

The OPERA detector was located at the Gran Sasso underground laboratory (LNGS) in Italy at $3800 \mathrm{~m}$ water-equivalent (m.w.e.) depth, and it has also been used as an observatory for $\mathrm{TeV}$ muons produced by cosmic rays in the atmosphere. In particular, the measurement of the single muon flux modulation and of its correlation with the seasonal variation of the atmospheric temperature was performed.

\section{The CNGS beam and the OPERA detector}

The CNGS (CERN Neutrinos to Gran Sasso) beam [2] was produced by accelerating protons to $400 \mathrm{GeV} / \mathrm{c}$ with the CERN Super Proton Synchrotron (SPS). These protons were ejected with a kicker magnet towards a $2 \mathrm{~m}$ long graphite target in two extractions, each lasting $10.5 \mu$ s and separated by $50 \mathrm{~ms}$. Each CNGS cycle in the SPS was $6 \mathrm{~s}$ long. Secondary mesons with positive charge were focused by a magnetic horn and reflector, each followed by a helium bag to minimise the interaction probability of the mesons. These mesons decayed in flight, mainly into neutrinos and muons, in a $1000 \mathrm{~m}$ long evacuated tunnel. The different components of the CNGS beam line are shown in Figure 1.

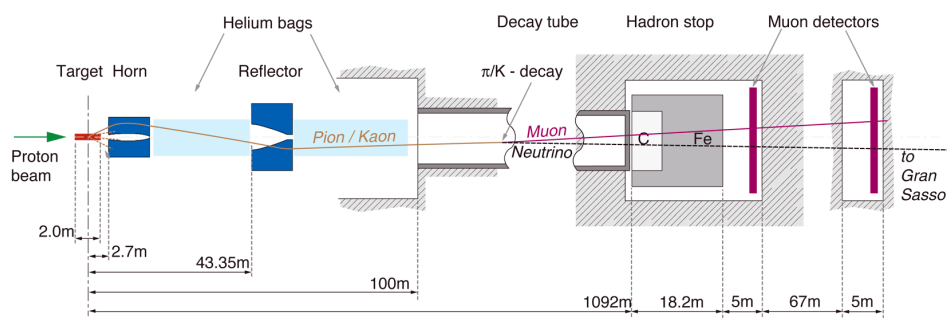

Figure 1: Layout of the CNGS beam line.

The beam consisted mainly of $v_{\mu}$ with a mean energy of about $17 \mathrm{GeV}$, with a $\bar{v}_{\mu}$ contamination of $2.1 \%$ and $v_{e}+\bar{v}_{e}$ contamination of $\sim 0.9 \%$. The prompt $v_{\tau}$ contamination was negligible. Using the SPS in a shared mode between fixed target experiments and the LHC, a total intensity of $\sim 1.8 \times 10^{20}$ protons on target (pot) was delivered to CNGS from June 2008 to December 2012.

The OPERA detector [3] was located at the LNGS laboratory, $730 \mathrm{~km}$ away from the SPS accelerator. Figure 2 shows a picture of the OPERA detector which was $20 \mathrm{~m}$ long with a cross section of about $8 \times 9 \mathrm{~m}^{2}$ and composed of two identical parts called supermodules (SM). Each SM had a target section and a muon spectrometer.

The target section was composed of vertical light supporting steel structures, called walls, interleaved with 31 double layered planes of an electronic Target Tracker (TT) detector. Each TT layer consisted of $2567 \mathrm{~m}$ long, $10.6 \mathrm{~mm}$ thick, and $26.3 \mathrm{~mm}$ wide scintillator strips read on both sides using Wavelength Shifting (WLS) fibres and multi-anode photo-multipliers (PMT). The main goals of the TT detector were to provide a trigger for the neutrino interactions and an efficient event pattern recognition. Together with the magnetic spectrometer, the TT detector allowed a clear classification of the $v$ events, and a precise localisation of the neutrino interaction vertex. It was also used as a calorimeter for the event analysis. The TT spatial resolution reached $\sim 0.8 \mathrm{~cm}$ and its efficiency was of $\sim 99 \%$ for a minimum ionizing particle. 


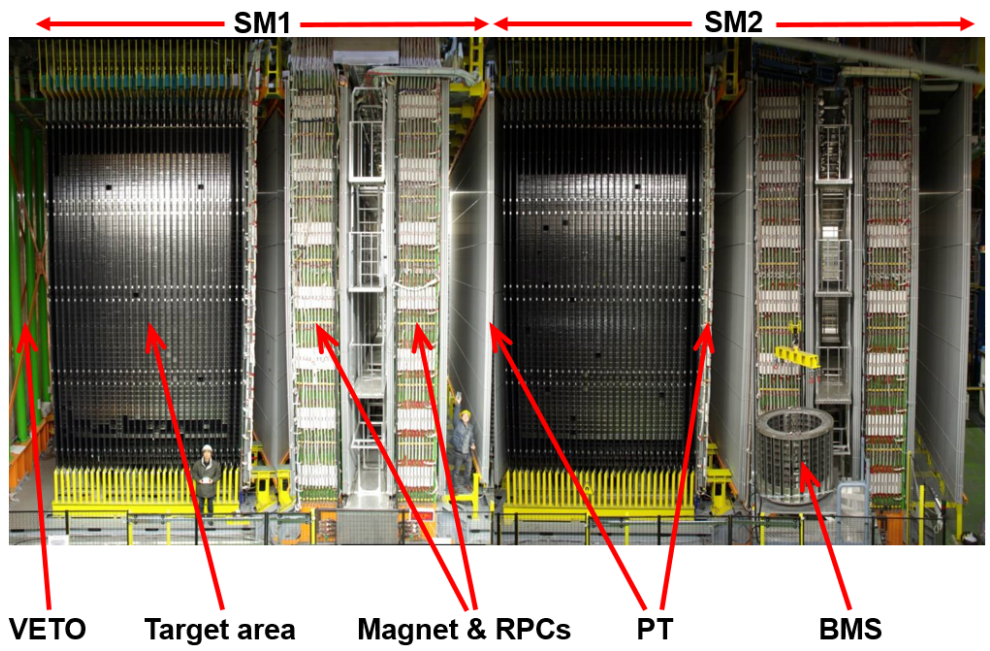

Figure 2: General view of the OPERA detector: two identical supermodules (SM1 and SM2), each including a target area, a magnet, and a system of resistive plane chambers (RPC) and drift tubes $(\mathrm{PT})$. The target area contained $\sim 75000$ bricks located between the planes of the Target Tracker (TT). The neutrino beam entered from the left. The VETO system was used for selecting neutrino events originating from the target area. The extraction of the bricks from the detector was done with a robotic Brick Manipulating System (BMS).

The walls in each SM contained $\sim 75000$ basic target detector units, called "bricks", with mass of $8.3 \mathrm{~kg}$ and size of $12.7 \times 10.2 \times 7.9 \mathrm{~cm}^{3}$ and where stacks of 56 lead sheets $1 \mathrm{~mm}$ thick interleaved with 57 plates of nuclear emulsion. This structure provided a massive target coupled to a very precise tracker, as well as standalone detectors to measure electromagnetic showers and charged particle momenta using multiple Coulomb scattering in lead [4]. Track segments were reconstructed using automated microscopes with angle and position resolutions of $2 \mathrm{mrad}$ and $0.21 \mu \mathrm{m}$, respectively. Downstream of each brick, an emulsion film doublet, called Changeable Sheet (CSd) was attached in a separate plastic envelope. The CS was detached from the brick for analysis to confirm and locate the tracks produced in neutrino interactions.

The spectrometer allowed a determination of the charge and momentum of crossing muons by measuring their curvature in a bipolar magnet made of 990 tons of iron, and providing a $1.53 \mathrm{~T}$ magnetic field transverse to the neutrino beam axis. Each spectrometer was equipped with six vertical planes of drift tubes (Precision Tracker, PT) together with 22 planes $\left(8 \times 8 \mathrm{~m}^{2}\right)$ of resistive plane chambers $(\mathrm{RPC})$ reaching a spatial resolution of $1 \mathrm{~cm}$ and an efficiency of $\sim 96 \%$. The PT planes were composed of 4 staggered layers of 168 aluminium tubes, $8 \mathrm{~m}$ long with a $38 \mathrm{~mm}$ outer diameter. The spatial resolution of this detector was better than $500 \mu \mathrm{m}$. The physics performance of the complete spectrometer reduced the charge confusion to less than $0.5 \%$ and gave a momentum resolution better than $20 \%$ for momenta less than $25 \mathrm{GeV}$. The muon identification efficiency reached $95 \%$ after adding the TT information for the cases where the muons stopped inside the target. 


\section{Event Analysis}

The OPERA event analysis, detailed in [5], proceeds in several steps starting by the acquisition of events generating a trigger in the electronic detectors in time coincidence with the CNGS spills. An on-line filtering is applied to keep the events with sufficient number of hits in the TT planes and to remove background from random noise. Using the TT and spectrometer information the events are reconstructed and classified as CC-like (" $1 \mu$ ") or neutral current (NC) like (" $0 \mu$ ").

Table 1: Summary of the collected data sample, the number of beam days and number of $v$ interactions fully reconstructed in the target during the 5 years of run.

\begin{tabular}{cccc}
\hline year & pot & beam days & $v$ interactions \\
\hline 2008 & $1.74 \times 10^{19}$ & 123 & 1757 \\
2009 & $3.53 \times 10^{19}$ & 155 & 3590 \\
2010 & $4.09 \times 10^{19}$ & 187 & 3932 \\
2011 & $4.75 \times 10^{19}$ & 243 & 4311 \\
2012 & $3.86 \times 10^{19}$ & 257 & 3281 \\
\hline Total & $17.97 \times 10^{19}$ & 965 & 16871 \\
\hline
\end{tabular}

During the CNGS running years $(2008-2012)$ a total of $17.97 \times 10^{19}$ pot was collected by OPERA which was $20 \%$ less than what was foreseen in the experiment proposal [1]. With a trigger efficiency of $99 \%$, a sample of 106422 on-time events was recorded: about $60 \%$ were from neutrino interactions in the rock surrounding the detector and about $20 \%$ were interactions occurring in the spectrometer. The remaining $20 \%$ yielded 19505 contained events of which $86 \%$ were fully reconstructed in the target. Table 1 summarises the characteristics of the collected data sample during the five CNGS runs.

For the events where the neutrino interaction occurred in the emulsion target, a brick finding algorithm based on the energy deposition in the TT strips and on muon track information (if available), was applied to define a 3D probability map for the brick vertex localisation. The identified brick was then extracted using automated brick manipulators located on each side of the detector.

The CSd films were readout ("scanned") by automated optical microscopes to confirm the vertex probability in the corresponding brick. In case of positive feedback, the emulsions in the brick were developed and sent to emulsion scanning laboratories in Europe and in Japan, that hosted high speed automated optical microscopes. This last step was the start of the detailed event analysis for the neutrino vertex location and the $\tau$ decay kink topology search in the vertex region.

In order to optimise the search for $v_{\mu} \rightarrow v_{\tau}$ oscillation, the scanning strategy evolved with time. In the 2008 and 2009 runs analysis, a conservative approach was used to get confidence on the detector performance: no kinematical cuts were applied, leading to a slow analysis speed with a signal/noise ratio not optimized. For the 2010, 2011, and 2012, kinematical selections were applied: a muon momentum of less than $15 \mathrm{GeV} / \mathrm{c}$, the most probable brick analyzed for all events before moving to the other ones, and the analysis of the $0 \mu$ events was anticipated to increase in a first phase the ratio between efficiency and analysis time. 
The vertex location was performed by following back the predicted tracks from the CSd films inside the brick taking into account multiple Coulomb scattering and measurement error effects. This "scanback" procedure was stopped when the track was not seen in three consecutive films. A detailed volume scan was then performed around the stopping point defined by a transverse area of $1 \mathrm{~cm}^{2}$ for 5 films upstream and 10 films downstream of it. After rejecting short and passing through tracks, tracks pointing to an interaction vertex were searched for in the corresponding volume. The mean efficiency to locate a neutrino interaction vertex was about $74 \%$ for CC-like events and $48 \%$ for NC-like events, compatible with the expected values from the Monte Carlo simulation [5].

Once a vertex had been identified, a "decay search" procedure aiming at detecting the decay topologies of $\tau$ leptons or charm hadrons was performed. A decay signature could be associated to a daughter track with a large impact parameter or with a significant kink with respect to a primary track. The number of decay searched events in the full data set amounted to 6785 .

$$
4 v_{\mu} \rightarrow v_{\tau}
$$

The were three main sources of background in the search for $v_{\tau}$ appearance: the $v_{\mu} \mathrm{CC}$ charm production ( $4 \%$ of $\mathrm{CC}$ ) with missing or mis-identified primary muon, the nuclear interactions of hadrons ( $0.2 \%$ of $\mathrm{NC}$ ), and the large angle coulomb scattering (LAS) of muons.

The kinematical variables used to reduce the background sources were the flight length, the total transverse momentum of $\tau$ daughters with respect to the $\tau$ direction, the missing transverse momentum at the primary vertex and the $\varphi$ angle of the $\tau$ with respect to the hadronic shower in the beam transverse plane.

\subsection{Minimum bias analysis}

Applying the topological and kinematical selection criteria described in [5] five $v_{\tau}$ candidate events were observed in the analysed data sample with an expected background of 0.25 dominated by the charm in the electron, muon and 3-prong decay channels.

Table 2: Expected signal and background events in the data sample for the minimum bias analysis.

\begin{tabular}{lccc}
\hline Channel & Exp. Background & Exp. Signal & Observed \\
\hline$\tau \rightarrow 1 h$ & $0.04 \pm 0.01$ & $0.52 \pm 0.10$ & 3 \\
$\tau \rightarrow 3 h$ & $0.17 \pm 0.03$ & $0.73 \pm 0.14$ & 1 \\
$\tau \rightarrow \mu$ & $0.004 \pm 0.001$ & $0.61 \pm 0.12$ & 1 \\
$\tau \rightarrow e$ & $0.03 \pm 0.01$ & $0.78 \pm 0.16$ & 0 \\
\hline Total & $0.25 \pm 0.05$ & $2.64 \pm 0.53$ & 5 \\
\hline
\end{tabular}

The numbers of expected signal and background events were estimated from the simulated CNGS flux [6]. The expected detectable signal events in the $0 \mu$ events and $1 \mu$ samples were obtained using the reconstruction efficiencies and the $v_{\tau}$ event rate in the flux normalised to the detected $v_{\mu}$ interactions. A similar normalisation procedure was also used in the background expectation. The details of the signal and background estimation are described in [5]. 
The expected numbers of $v_{\tau}$ events for each decay channel were computed assuming $\Delta m_{32}^{2}=2.44 \times 10^{-3} \mathrm{eV}^{2}[7]$ and maximal mixing (see Table 2). The probability for the 5 candidate events to be explained by background fluctuation was estimated to be $1.1 \times 10^{-7}$. From the latter the no-oscillation hypothesis was excluded at 5.1 $\sigma$ [8].

\subsection{Extended analysis}

After demonstration that $v_{\mu}$ do oscillate in $v_{\tau}$, a new goal has been set: estimate $\Delta m_{23}^{2}$ in appearance mode and the $v_{\tau}$ cross-section $\left(\sigma_{v_{\tau}}\right)$, so far measured only by the DONuT experiment, that however could not distinguish $v_{\tau}$ from $\bar{v}_{\tau}$. To reduce the statistical error in these values estimation, a new strategy has been defined with looser kinematical cuts and a multivariate analysis.

Table 3: Comparison between the selection cuts used to establish the $v_{\tau}$ appearance (PREV.) and the extended ones (NEW). Cuts marked with $\star$ are not applied for Quasi-Elastic events. $p_{2 r y}^{T}$ cut is 0.3 in the presence of $\gamma$ particles associated to the decay vertex.

\begin{tabular}{|c|c|c|c|c|c|c|c|}
\hline \multirow{2}{*}{ Variable } & \multicolumn{2}{|c|}{$\tau \rightarrow 1 h$} & \multicolumn{2}{|c|}{$\tau \rightarrow 3 h$} & \multicolumn{2}{|c|}{$\tau \rightarrow \mu$} & $\tau \rightarrow e$ \\
\hline & PREV. & NEW & PREV & NEW & PREV. & NEW & PREV. NEW \\
\hline$z_{\text {dec }}(\mu m)$ & {$[44,2600]$} & $<2600$ & \multicolumn{2}{|c|}{$<2600$} & {$[44,2600$} & $<2600$ & \multirow{2}{*}{$\begin{array}{l}<2600 \\
>0.02\end{array}$} \\
\hline$\theta_{\text {kink }}(\mathrm{rad})$ & \multicolumn{2}{|c|}{$>0.02$} & $<0.5$ & $>0.02$ & $>$ & & \\
\hline$p_{2 r y}(G e V / c)$ & $>2$ & $>1$ & $>3$ & $>1$ & & & {$[1,15] \quad>1$} \\
\hline$p_{2 r y}^{T}(G e V / c)$ & $>0.6(0.3)$ & $>0.15$ & & & $>0.25$ & $>0.1$ & $>0.1$ \\
\hline$p_{m i s s}^{T}(G e V / c)$ & $<1 \star$ & / & $<1 \star$ & / & & & / \\
\hline $\begin{array}{c}\phi_{l H}(\mathrm{rad}) \\
m, m_{\min }\left(\mathrm{GeV} / \mathrm{c}^{2}\right)\end{array}$ & $>\pi / 2 \star$ & 1 & $\begin{array}{l}>\pi / 2 \star \\
{[0.5,2]}\end{array}$ & / & & & / \\
\hline
\end{tabular}

The extended selection criteria (NEW) are shown in the Table 3, compared with the previous selection criteria (PREV.) defined in [5].

Table 4: Expected signal and background events in the data sample for the extended analysis.

\begin{tabular}{lccc}
\hline Channel & Exp. Background & Exp. Signal & Observed \\
\hline$\tau \rightarrow 1 h$ & $1.43 \pm 0.41$ & $2.96 \pm 0.59$ & 6 \\
$\tau \rightarrow 3 h$ & $0.53 \pm 0.12$ & $1.83 \pm 0.37$ & 3 \\
$\tau \rightarrow \mu$ & $0.03 \pm 0.01$ & $1.15 \pm 0.23$ & 1 \\
$\tau \rightarrow e$ & $0.03 \pm 0.007$ & $0.84 \pm 0.17$ & 0 \\
\hline Total & $2.0 \pm 0.5$ & $6.8 \pm 1.4$ & 10 \\
\hline
\end{tabular}

With the new analysis strategy more signal and background events are expected, as summarized in the Table 4.

OPERA is the first experiment that can measure $\Delta m_{23}^{2}$ in appearance mode. The result of the measurement based on the $5 v_{\tau}$ signal candidates that passed the minimum bias cuts (see 4.1) was given in [8]. The updated result based on the 10 observed events, with 6.8 signal and 2.0 background expected events, has been evaluated. Assuming full mixing 


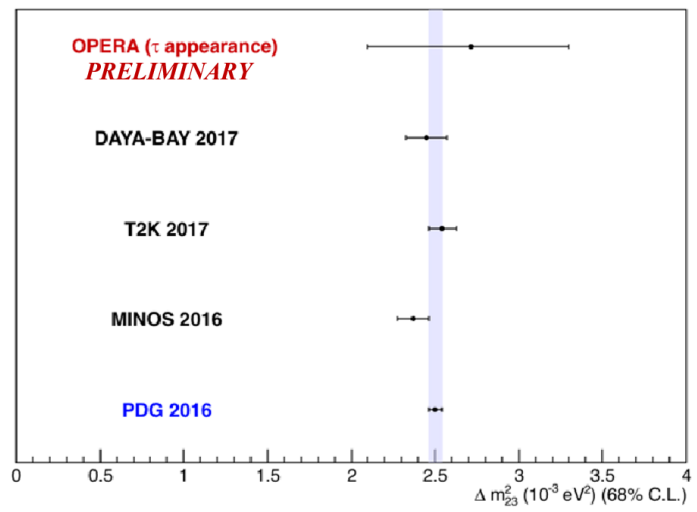

Figure 3. Comparison of $\Delta m_{23}^{2}$ from different experiments at $68 \%$ C.L.

the $68 \%$ C.L. interval for $\Delta m_{23}^{2}$ is $[2.1,3.3] \times 10^{-3} \mathrm{eV}^{2}$. The result has been compared with the one given by PDG 2016 [9], which is $(2.50 \pm 0.13) \times 10^{-3} \mathrm{eV}^{2}$, and the ones found in disappearance mode by other experiments such as DAYA-BAY, T2K and MINOS. The comparison with $68 \%$ C.L. errors is shown in Figure 3. The measurement performed is in agreement with the PDG value within $1 \sigma$.

In order to evaluate the $v_{\tau}$ cross-section, $\Delta m_{23}^{2}$ has been fixed, since the OPERA experiment is sensitive to the product of $\Delta m_{23}^{2}$ and $\sigma_{v_{\tau}}$. Assuming $\Delta m_{23}^{2}=2.50 \times 10^{-3} \mathrm{eV}^{2}$ and the maximal mixing $\sin ^{2} 2 \theta_{23}=1$,

$$
\sigma_{v_{\tau} \text { meas }}^{0}=\left(8_{-3}^{+4}\right) \times 10^{-39} \mathrm{~cm}^{2} \mathrm{GeV}^{-1} \text { at } 68 \% \text { C.L. }
$$

The measurement performed is in agreement with the Standard Model value [9], which is $6.7 \times 10^{-39} \mathrm{~cm}^{2} \mathrm{GeV}^{-1}$, within $1 \sigma$.

$5 v_{\mu} \rightarrow v_{e}$

Thanks to the dense brick structure and the high granularity provided by the nuclear emulsions, the OPERA detector was also suited for electron and $\gamma$ detection. This allowed to study the $v_{e}$ CC interactions and therefore to search for $v_{e}$ appearance from $v_{\mu} \rightarrow v_{e}$ oscillations.

A dedicated procedure was applied to $0 \mu$ events aiming at identifying "shower hints" from track multiplicity in the CSd. An additional scanning of the volume extending from the most downstream film up to the interaction vertex was performed in order to reconstruct electromagnetic showers. Events with a shower initiated by a single track emerging from the primary vertex were classified as $v_{e}$ candidates. The first result corresponding to $5.3 \times 10^{19}$ pot was published in [10]. The search has been extended to the whole data set (a more than twofold increase in statistics) yielding $35 v_{e}$ candidates. The expected number of $v_{e} \mathrm{CC}$ interactions due to the intrinsic beam contamination is $31 \pm 3$. Background events amount to $1.3 \pm 0.5$. They arise from misidentified $\pi_{0}$ in $v_{\mu}$ interactions without a reconstructed muon and $v_{\tau}$ CC interactions with $\tau$ decaying into an electron. In the whole energy range $2.7 \pm 0.3$ oscillated $v_{e} \mathrm{CC}$ events are expected assuming $\sin ^{2} \theta_{13}=0.0210, \sin ^{2} \theta_{23}=0.51$, $\Delta m_{31}^{2}=2.44 \times 10^{-3} \mathrm{eV}^{2}, \delta C P=0$, and neglecting matter effects. In conclusion, the number of observed events is compatible with the 3 -flavour oscillation model.

Figure 4 shows the reconstructed energy distribution of these events compared to the expected reconstructed energy spectra from the various sources described above. 


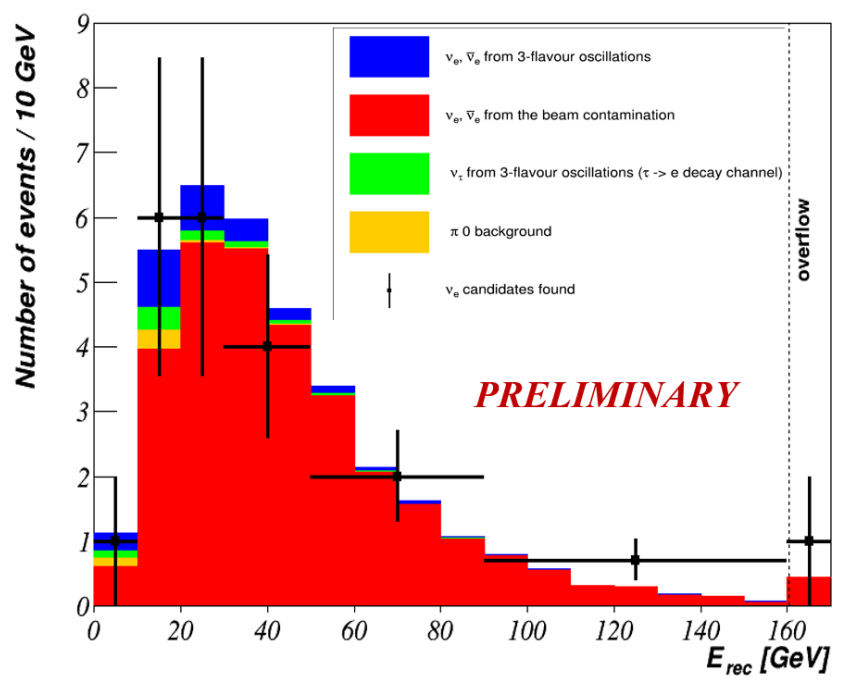

Figure 4. Distribution of the reconstructed energy of the $v_{e}$ events, and the expected spectra from the various sources, normalized to the measured $v_{\mu} 0 \mu$ sample.

\section{Search for sterile neutrino mixing}

The obtained results on $v_{\tau}$ and $v_{e}$ appearance can be interpreted in the context of the 3 or $3+1$ neutrino models deriving limits on oscillations induced by a massive sterile neutrino. The appearance probability is modified by the addition of oscillation and interference terms from the new sterile state.
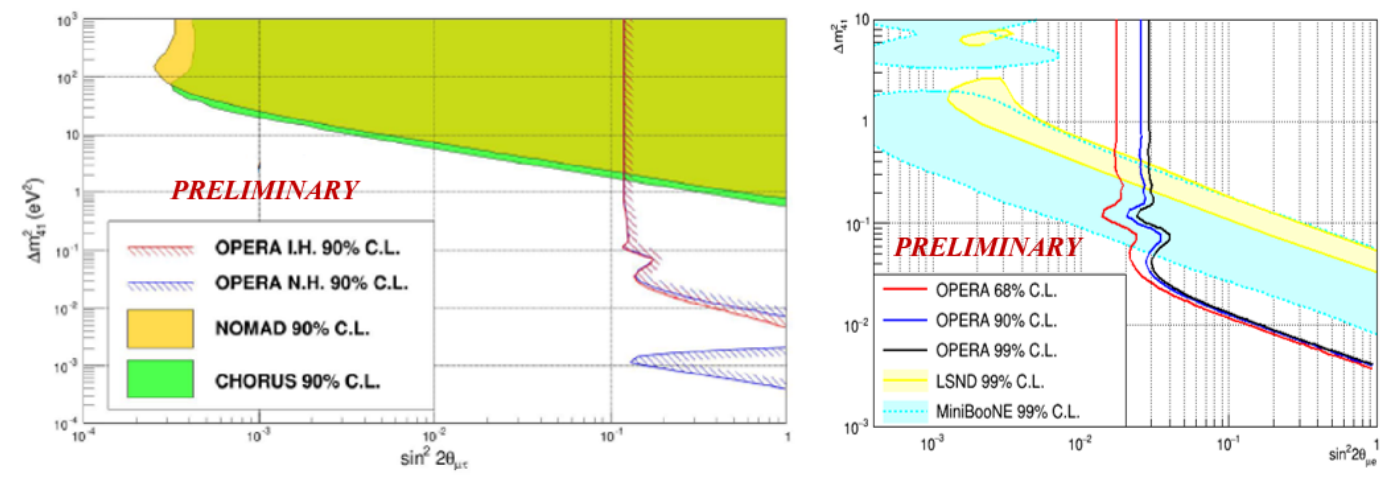

Figure 5: The exclusion plots for the parameters of the non-standard $v_{\mu} \rightarrow v_{\tau}$ (left) and $v_{\mu} \rightarrow v_{e}$ (right) oscillations in the $3+1$ neutrino models.

A detailed description of the analysis and the results obtained by OPERA with limited statistics were given in [10] (for the 2 neutrino mixing scheme) and in [11] (for the $3+1$ neutrino model). Here we present preliminary updated results for the $3+1$ neutrino model obtained with the final data samples described in 4.1 and 5. In Figure 5 (left and right) the OPERA exclusion contours are shown together with existing limits from previous experiments. 


\section{Annual modulation of the cosmic muon rate}

Here the preliminary results of the measurement of the single muon flux modulation and of its correlation with the seasonal variation of the atmospheric temperature are reported. The analysis is based on TT and RPC data acquired during about five years from January 2008 to December 2012. The TT systems were operative during most of the time in the considered years, while the RPCs had a lower run-time, being operative only during the CNGS beam. To compare periods with and without RPC data acquired in addition to the TT, scale factors have been evaluated in those days when both systems were in acquisition. About $1950 \mu$ /day have been reconstructed by the TT alone, reaching the value of $3400 \mu$ /day when also the RPC systems were active.

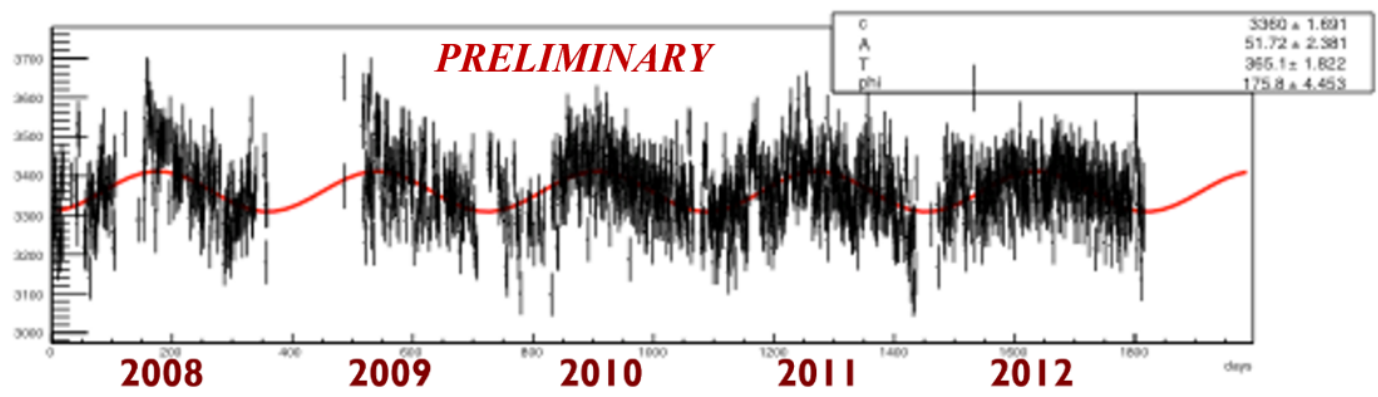

Figure 6: Single muon rate measured by the OPERA detector from January 2008 to March 2013.

In Figure 6 the single muon cosmic ray flux measured by OPERA from January 1st 2008 (day 1 in the plot) to March 2013 is shown. The flux has been fitted with a function $I_{\mu}(t)=I_{\mu}^{0}+\delta I_{\mu} \cos \left(2 \pi / T \times\left(t-t_{0}\right)\right)$. The parameters obtained from the fit are the following ones: $I_{\mu}^{0}$ is the mean event rate, $\delta I_{\mu}=(1.54 \pm 0.07) \%$ is the amplitude of the component modulated with period $T=(365 \pm 2)$ days and phase $t_{0}=(175 \pm 4)$ days.

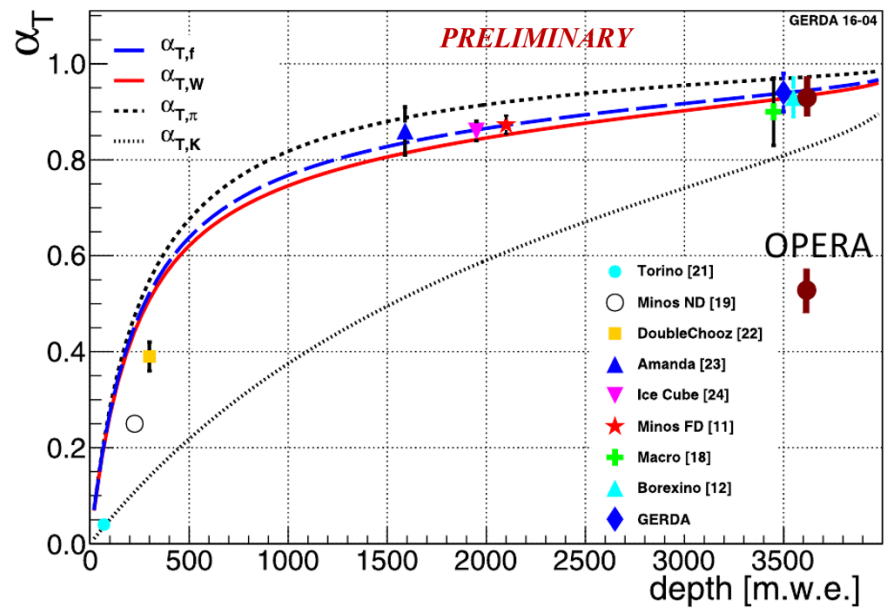

Figure 7. Effective temperature coefficient $\alpha_{T}$ vs depth as measured by different underground experiments. The red line is the value predicted assuming muon production from pions and kaons; the dashed lines correspond to the single production mechanisms. 
The muon flux, produced mainly by pions and kaons decays, has a dependency with the atmosphere's effective temperature $T_{e f f}$. To measure the atmospheric temperature modulation, the data from the European Center for Medium-range Weather Forecasts (ECMWF) have been used. The effective atmospheric temperature at the coordinates of the LNGS underground halls has been estimated using the modelization, reported in [12]. The "effective temperature coefficient", $\alpha_{T}=\delta I_{\mu} / \delta T_{\text {eff }}$, has been found to be $0.94 \pm 0.04$. The obtained value is in agreement with the predictions for the LNGS site and with the results of other experiments (see Figure 7).

\section{Conclusions}

The OPERA experiment took data for 5 years between 2008 and 2012 and the $v_{\mu} \rightarrow v_{\tau}$ oscillation was observed with a confidence level of $5.1 \sigma$ outreaching the proposal expectations and allowing to claim discovery of $v_{\tau}$ appearance. In order to estimate $\Delta m_{23}^{2}$ in appearance mode and $v_{\tau}$ cross-section with reduced statistical uncertainty an analysis using a selection with released cuts and multivariate techniques is being performed.

The search for $v_{\mu} \rightarrow v_{e}$ oscillation has been extended to the whole data sample. The number of events observed is in agreement with the expected background and the standard oscillation signal.

The results on $v_{\mu} \rightarrow v_{\tau}$ and $v_{\mu} \rightarrow v_{e}$ searches are being used to constrain the parameter space of oscillations induced by a massive sterile neutrino.

The preliminary results of the measurement of the single muon flux modulation and of its correlation with the seasonal variation of the atmospheric temperature have been presented.

\section{References}

[1] Guler M. et al. (OPERA Collaboration), CERN-SPSC-2000-028, CERN-SPSC-P-318, LNGS-P25-00.

[2] CNGS Project, http://proj-cngs.web.cern.ch/proj-cngs/

[3] Acquafredda R. et al. (OPERA Collaboration), JINST 4, P04018 (2009).

[4] Agafonova N. et al. (OPERA Collaboration), New J. Phys. 14, 013026 (2012).

[5] Agafonova N. et al. (OPERA Collaboration), JHEP 11, 036 (2013), Erratum-ibid. 04, 014 (2014).

[6] CNGS Neutrino Flux Calculations, http://www.mi.infn.it/ psala/Icarus/cngs.html

[7] Olive K.A. et al. (Particle Data Group), Chin. Phys. C 38, 090001 (2014).

[8] Agafonova N. et al. (OPERA Collaboration), Phys. Rev. Lett. 115, 121802 (2015).

[9] Patrignani C. et al. (Particle Data Group), Chin. Phys. C 40, 100001 (2016).

[10] Agafonova N. et al. (OPERA Collaboration), JHEP 07, 004 (2013), Addendum: 07, 085 (2013).

[11] Agafonova N. et al. (OPERA Collaboration), JHEP 06, 069 (2015).

[12] Grashorn E.W. et al., Astropart.Phys. 33, 140(2010). 